\title{
Anpassen oder aufklären? Finanzerziehung und sozioökonomische Bildung'
}

\author{
*reinhold.hedtke@uni-bielefeld.de, Fakultät für Soziologie, Universität Bielefeld
}

eingereicht am: 17.08.2018, akzeptiert am: 02.11.2018

Finanzbildung genießt große Aufmerksamkeit. In der Öffentlichkeit wird sie als dringendes Desiderat präsentiert; Finanzindustrie und Bildungspolitik fordern unisono ihre Institutionalisierung an Schulen. Junge Menschen und Erwachsene werden oft als Finanzanalphabet/innen beschimpft. Was aber macht die Beschäftigung mit Finanzthemen zur Bildung? Was kennzeichnet einen sozioökonomisch aufgeklärten Zugang zum Themenfeld Finanzen? Das von vielerlei Akteur/innen bespielte Feld der Finanzbildung bringt reichlich Anschauungsmaterial für die Klärung dieser Fragen hervor. Der vorliegende Beitrag verwendet es, um die Unterscheidung zwischen sozioökonomischer Bildung und funktionalistischer Finanzausbildung herauszuarbeiten. Dazu analysiert er im ersten Schritt beispielhaft das Framing, mit dem das Desiderat „Finanzbildung “ inhaltlich programmiert und politisch propagiert wird. In einem zweiten Schritt diskutiert er die Differenz von Finanzlernen und Bildung zum Themenkomplex Finanzen, die interessenpolitischen Einflüsse und die sozialwissenschaftlichen Grundlagen von Finanzerziehung. Der dritte Schritt dient der Analyse der politischen Dimension von Finanzausbildung im Kontext von marktfundamentalistischem Anpassungslernen und finanzieller Ungleichheit. Der Beitrag schließt mit einem Plädoyer für Bildung als Bürge der Freiheit.

Keywords: Finanzbildung, sozioökonomische Bildung, Ungleichheit, Finanzindustrie, Entpolitisierung

\section{To adjust or to enlighten? Financial education and socio-economic education}

Financial education enjoys great attention. In public, it is presented as an urgent desideratum; the financial industry and education policy unanimously call for their institutionalisation in schools. Young people and adults are often referred to as financial illiterates. What, however, turns learning about financial topics into education? What characterizes a socioeconomically informed approach to finance? The field of financial education is used by many actors and provides ample illustrative material for tackling these questions. This article uses it to highlight the distinction between socioeconomic education and functionalist financial education. In the first step, an exemplarily analysis of the framing is done, which is used to programme the desideratum "financial education" in terms of content, and to propagate it politically. In a second step, it discusses the difference between financial education and learning about the subject of finance, stakeholders' political influence, and the social science fundamentals of financial education. In the third step, an analysis of the political dimension of financial education in the context of market-fundamentalist, adaptation-oriented learning, and financial inequality is conducted. The article concludes with a plea for education as a guarantor of freedom.

Keywords: financial education, socio-economic education, inequality, finance, depoliticisation

\section{Framing von Bildung}

In einem Artikel in Die Presse berichtet Beate Lemmer über eine Studie der Organisation für wirtschaftliche Zusammenarbeit und Entwicklung (OECD) und ihres Internationalen Netzwerks für Finanzbildung (INFE). Das INFE hat nach eigenen Angaben rund 240 öffentliche Akteure wie Zentralbanken, Finanz- ministerien und Bildungsministerien als Mitglieder (OECD 2017). Die Studie fand in Form von fragebogengestützten Interviews statt. Die Befragung ist ein Instrument aus dem klassischen Werkzeugkasten, aus dem sich Finanzindustrie, Wirtschaftsverbände

Der Autor dankt zwei anonymen Gutachterinnen oder Gutachtern für ihre wertvollen Hinweise zur Überarbeitung dieses Beitrags. 
und Bildungspolitik häufig bedienen. Mit ihr kann man Berichterstattung erzeugen. Die OECD-Studie referiert unter anderem, dass in den G20 Ländern die meisten Erwachsenen zwar Zinsen verstehen, nicht jedoch, wie sich der Zinseszins auswirkt.

Die Journalistin stellt ihren Beitrag unter die Überschrift „Zinseszins wird oft nicht verstanden“ und beginnt ihn mit der Feststellung, es sei um „die ,finanzielle Alphabetisierung' der Erwachsenen in den wichtigen Industrie- und Schwellenländern nicht allzu gut bestellt" (Lemmer 2017). Dabei schneidet Österreich ganz ordentlich ab, liegt es doch mit 44 Prozent richtigen Antworten zur Zinseszins-Testfrage leicht über dem Durchschnitt der 30 Teilnehmerländer, zugleich aber etwas unter dem OECD-Mittel (OECD 2016: 23-24). Das hindert aber etwa eine/n Sparkassendirektor/in oder eine/n Wirtschaftspädagoge/in nicht daran, in den Chor der allfälligen Klagen über zu wenig Wissen einzustimmen (Greimel-Fuhrmann et al. 2016: 260). Aus dem Ausland beobachtet man mit Amüsement, wie dem Land mit einer der weltweit niedrigsten Armutsraten der dringende Bedarf attestiert wird, das allgemeine Verständnis von Geld und finanziellen Angelegenheiten zu verbessern (Willis 2018: 83).

\subsection{Finanzielle oder mathematische Bildung?}

Das für die Fachdidaktik Interessante und für die Bildungspolitik Relevante ist nicht dieser Befund selbst, sondern seine kommunikative Rahmung. Tatsächlich erhoben wurden unter anderem Kenntnisse der Zinseszinsrechnung. Dieses Wissen wird üblicherweise der mathematischen Bildung zugerechnet und steht auf Mathematik-Lehrplänen. Seine Messung gehört in den Kontext von allgemeinen Wissens- und Kompetenzstudien wie TIMSS oder PISA, auch wenn PISA inzwischen politische Vorgaben, wie die der OECD aufgreift und es in einem separaten Sektor, „financial literacy", ausweist. Renten- und Investitionsrechnung sind bekanntlich Teilgebiete der Finanzmathematik, die mathematische Konzepte wie Folgen und endliche Reihen auf finanzielle Fragestellungen anwendet. Diese Konzepte stehen in den Lehrplänen für den Unterrichtsgegenstand Mathematik, in Österreich zum Beispiel für die Allgemeinbildenden Höhere Schulen (AHS) und die Neue Mittelschule (NMS). Es sind in erster Linie Mathematik-Lehrkräfte, die über die fachwissenschaftlich-fachdidaktische und unterrichtspraktische Kompetenz dafür verfügen, nicht Lehrkräfte der Wirtschaftswissenschaften oder der Wirtschaftspädagogik. Ein schulisch typisches Format für mathematische Anwendungen, etwa in der Zinsrechnung, sind Textaufgaben.

Fehlt es am Können bei Zinsen und Zinseszinsen, lautet die naheliegende Forderung also, man braucht besseren oder mehr oder praxisnäheren Mathematikunterricht. Stattdessen unterzieht man den zitierten Befund einem politischen und ideologischen Reframing, um ihn in der öffentlichen Debatte gezielt einsetzen zu können (vgl. Wehling 2016: 42-67). Man definiert das Mathematikdefizit kurzerhand zum Indiz für eine unzureichende „Finanzbildung“ um. Was wird assoziiert? Wer die Zinseszinsrechnung nicht beherrscht, ist ungebildet, und er hat seine Finanzen nicht im Griff. Das läuft auf eine Falschetikettierung und sachfremde politische Instrumentalisierung hinaus. Die Beispiele dafür häufen sich in den letzten Jahren.

Kompetenzen, die im Kern die Beherrschung und den Transfer mathematischer Konzepte betreffen, werden sprachlich aufgewertet und überhöht, und als ein Teil von „Finanzbildung" ausgewiesen, statt von Finanzwissen zu sprechen. Zugleich wird der universale Anspruch von Modellierung in der mathematischen Bildung, die ganz selbstverständlich alltägliche Fragestellungen aus unterschiedlichen Anwendungsfeldern einschließt, bewusst ignoriert und die spezielle Anwendung auf Geldanlagen verabsolutiert. Die Faktenlage wird verdrängt: In der Schulmathematik steht diese Art von "Finanzbildung“ seit Jahrzehnten auf dem Stundenplan. So simuliert man Defizite, die man für sachfremde politische Forderungen nutzen kann.

Die Willkür derartiger interessenpolitischer Reframings illustrieren mögliche Kopien dieser Strategie. Wer Probleme mit Raumkonzepten wie Quadratmeter und Kubikmeter hat und mit der Tilgungsrechnung, als Sonderfall der Rentenrechnung, nicht zurechtkommt, bräuchte dringend eine „Immobilienbildung". Physikalische Wissensdefizite bei der Berechnung beschleunigter Bewegungen, etwa am Beispiel „Anhalteweg“ (ein Thema aus der Mechanik), könnte man zur Diagnose einer mangelhaften Verkehrsbildung umformatieren und bildungspolitische Abhilfe fordern, am besten durch ein Schulfach „Verkehr"“. Weitere Beispiele ließen sich leicht ergänzen.

Bildungspolitisch konsequent umgesetzt würde dies eine heillose Zersplitterung der schulischen Bildung in eine Vielzahl von Sonderzwecken und isolierten Unterrichtsgegenständen bewirken. Die grundlegende mathematische Bildung, die immer auch die genannten Anwendungsfelder aufgreift, würde beschränkt zugunsten von Spezialbildungen ohne Verallgemeinerungs- und Transferpotenzial. Wer verantwortungsvolle Bildungspolitik am Interesse der Schüler/innen und am Interesse der Gesellschaft an kompetenten Bürger/innen orientiert, der muss das verhindern.

\subsection{Produzierte Wissenslücken}

Ähnliche Strategien wie beim politischen Projekt Finanzbildung stecken hinter den seit Jahren fortlaufend 
produzierten Belegen für ein angeblich mangelhaftes ökonomisches Wissen der Bevölkerung insgesamt oder der Jugendlichen und jungen Erwachsenen im Besonderen (Stieger \& Jekel 2018). Durch Tests definierte und aufgedeckte Wissenslücken werden für politische Forderungen nach einem Schulfach „Wirtschaft" instrumentalisiert.

Die Wissenstests sind inhaltlich meist fragwürdig bis wertlos. Erstens fehlt die Prüfung der fachlichen und praktischen Relevanz des Wissens, sodass nicht deutlich wird, ob die Wissenslücken Handlungsbedarf indizieren oder nicht. Zweitens verzichten die Entdecker/innen der Wissenslücken, die qua Testkonstruktion tatsächlich deren Produzent/innen sind, in der Regel darauf, Vergleichsgrößen heranzuziehen. Deshalb fehlen Informationen darüber, ob das erhobene wirtschaftliche Wissen ebenso lückenhaft ist wie das politische, rechtliche oder geografische, oder ob es viel schlechter oder sogar besser ausfällt. Kurz und zugespitzt: Macht man Wissenstests, erfährt man meist, was man sowieso schon zu wissen meint.

Deshalb sind solche Testergebnisse für eine seriöse Bildungspolitik und Bildungspraxis unbrauchbar, aber interessenpolitisch sehr nützlich. Die Kommunikationsstrategie besteht darin, den Verzicht auf Relevanzprüfung und domänenübergreifenden Vergleich mit der unablässigen Wiederholung einer Behauptung über einen langen Zeitraum hinweg zu kombinieren, die auf den Truth-Effekt zielt, nach dem immer wieder Berichtetes schließlich als wahr geglaubt wird (Kepplinger 2010: 219-222; Koch \& Zerback 2011). So erzeugt man den Glauben der Öffentlichkeit an den Eindruck, ökonomisches Wissen sei wichtig, aber mangelhaft und man müsse dringend etwas tun.

Forderungen nach mehr Finanzwissen werden oft mit Forderungen nach einem separaten Schulfach Wirtschaft und mehr Zeit für wirtschaftswissenschaftliche Lerninhalte verbunden. Um die Voraussetzungen dafür durchzusetzen, bekämpfen Interessengruppen in Deutschland mit bildungspolitischen und medialen Kampagnen die Integrationsfächer der politischen Bildung, die seit jeher Politik, Wirtschaft und Gesellschaft in einem Schulfach behandeln (Hedtke 2015). In Österreich richten sich die lobbyistischen Vorstöße bekanntlich gegen das Domänenmodell Geographie und Wirtschaft, das auf eine mehr als 55-jährige Tradition zurückblicken kann - und damit sogar ein bisschen älter ist als die sozioökonomische Bildung, die in der zweiten Hälfte der 1960er Jahre aufkam.

Besonders bemerkenswert erscheint die Einseitigkeit, mit der ausschließlich für die Teildomäne Wirtschaft ein eigenes Fach gefordert wird. Dagegen bleiben Forderungen aus, jeweils eigene Schulfächer für die Domänen Recht, Politik oder Gesellschaft, für Arbeit, Konsum oder Familie einzurichten. Diese Se- lektivität zugunsten von Wirtschaft hat eine zweifache Ursache: Dieses Fach ist im Interesse der Wirtschaftsverbände und ihrer Verbündeten in Medien, Wissenschaft und Bildungspolitik, und diese Akteur/innen verfügen über die monetären, medialen und machtförmigen Ressourcen dieses Thema auf der politischen und öffentlichen Agenda zu halten.

Zur Plausibilisierung der Forderung nach einem Separatfach Wirtschaft greift man auf Elemente des politisch-ideologischen Framings zurück. Zum einen arbeitet man mit dem Selektivitätseffekt (Wehling 2016: 42-45). Man fokussiert allein auf das Separatfach Wirtschaft und ignoriert die Lage der anderen Teildomänen und Fächer in der sozialwissenschaftlichen Domäne der Schule. Die negativen Nebenfolgen, die die Absonderung von Wirtschaft bei Dritten bewirkt, bleiben ausgeblendet. Die Selektionstechnik beim Framing greift beispielsweise auch dann, wenn Wirtschaftsdidaktiker/innen in Deutschland das „Desinteresse der Kultusministerkonferenz" an ökonomischer Bildung beklagen, und dies damit belegen, „dass sich Deutschland weder 2012 noch 2015 an der Erhebung der finanziellen Allgemeinbildung im Rahmen von Pisa beteiligt hat" (Retzmann 2016). Das ist Selektivität zwecks Irreführung, da Deutschland sich weder 2009 noch 2016 an der International Civic and Citizenship Education Study beteiligt hat (mit Ausnahme von Nordrhein-Westfalen in 2016). Ein spezifisches Desinteresse an ökonomischer Bildung kann man so nicht belegen.

Zum anderen verbreitet man durch fortlaufende Wiederholung und in vielen Varianten bei jeder Gelegenheit Behauptungen des Typs „Die Schule ist ein wirtschaftsfreier Raum“ (Retzmann 2016). Damit erzielt man den erwähnten Truth-Effekt und schafft einen sprachlichen Rahmen, der sich auf die weitere Wahrnehmung der Welt auswirkt (Wehling 2016: 32-34). Obwohl Behauptungen wie "wirtschaftsfrei“ oder "kaum oder gar keine ökonomische Bildung“ in die Abteilung „Desinformation und Propaganda“ fallen, weil sie empirisch - etwa mit Blick auf Fächer, Curricula, Berufsorientierung, Betriebspraktika oder Kooperationen Schule-Wirtschaft - schlicht falsch sind, werden sie häufig wiederholt und heftig beklagt.

\subsection{Fakten und Framing}

Was kann man gegen Falschinformation und Irreführung unternehmen? Man muss beharrlich an der entscheidenden Frage festhalten: Was ist wirklich der Fall? Dazu muss man eigene empirische Analysen durchführen und von Dritten vorgelegte Studien sorgfältig analysieren und ggf. kritisieren. Sandra Stieger und Thomas Jekel haben jüngst gezeigt, was eine wissenschaftlich solide Kritik aufdecken kann (Stieger \& Jekel 2018). 
Wie man das politisch-ideologische Framing der „Wirtschaftsferne“ mit der curricularen Faktenlage konfrontieren kann, illustriert eine aktuelle Studie zur Sekundarstufe I im Bundesland Nordrhein-Westfalen (Gökbudak \& Hedtke 2017). Sie analysiert den curricularen Stand der Dinge nach sieben Jahren Koalitionsregierung von SPD und Die Grünen. Die Studie erfasst für die Gesamtschule die beiden einschlägigen Pflichtfächer Politik und Wirtschaft, für die Realschule Politik und für das Gymnasium Politik/ Wirtschaft. Die Stundentafel der Gesamtschule sieht insgesamt sechs Wochenstunden Politik und 3,3 Wochenstunden Wirtschaft vor, darüber hinaus gibt es das obligatorische Schüler/innenbetriebspraktikum, das mindestens einem Unterrichtsumfang von zwei Wochenstunden entspricht und zweimal durchgeführt werden kann. Hinzu kommen zeitintensive Aktivitäten zur Berufsorientierung. Berücksichtigt man nur den regulären Unterricht und rechnet die Anteile obligatorischer Themenfelder im Curriculum der beiden Gesamtschulfächer zusammen, ergeben sich 15 Prozent gesellschaftliche Themen, 26 Prozent politische und 59 Prozent wirtschaftliche Themen. Im Fach Politik an der Realschule lauten die Anteile 23 zu 39 zu 38 Prozent, am Gymnasium im Fach Politik/ Wirtschaft 27 zu 32 zu 41 Prozent (Gökbudak \& Hedtke 2017, 9, 11).

Das politische Framing von ökonomischer Bildung mittels der Behauptung, die „Bildungs- und Schulpolitik vernachlässigt dieses Thema sträflich“ (Retzmannn 2016), ist empirisch falsch. Das gilt nicht nur für Nordrhein-Westfalen, sondern für alle Bundesländer, wie eine aktuelle Vollerhebung aller einschlägigen Curricula der Sekundarstufe I belegt (Weber 2018). Für das österreichische Modell sozioökonomischer Bildung, das traditionell auf die Integration von Geographie und Wirtschaft setzt, ist die Behauptung für die Allgemeinbildende Höhere Schule und die Neue Mittelschule ebenso falsch (Fridrich 2015, 2018; Fridrich \& Hofmann-Schneller 2017). In Österreich, wie in Deutschland, ist ein sozialwissenschaftliches Integrationsfach der Regelfall. In der deutschen Kultusministerkonferenz herrscht Konsens, dass (sozio)ökonomische Bildung Teil der gesellschaftswissenschaftlichen Domäne ist (KMK 2013/1972: 6, 2014/1993: 9). Auch in Österreich ist sie in einem „traditionell sozialwissenschaftlich angelegten Fach wie etwa Geographie und Wirtschaftskunde" integriert (Fridrich 2018: 104).

Es ist allgemein bekannt und trivial, dass die Existenz eines Schulfachs und der Unterricht für eine bestimmte Wissensdomäne nicht garantieren, dass die Schüler/innen dort einen befriedigenden Wissensstand erreichen und dauerhaft halten. Das gilt selbstverständlich auch für neu einzurichtende Schulfächer.
Auch verwundert es nicht, dass die Klagen über Wissenslücken von Lernenden Legion sind und sich seit Jahrhunderten wiederholen. Denn die meisten Menschen wissen in fast allen Domänen wenig bis nichts. Roland Reichenbach konstatiert, „im Vergleich zum Bereich der Inkompetenz, zu dem also, was wir alles nicht, kaum oder nur schlecht können, ist der Bereich der Kompetenz sehr klein. Der Bereich der Inkompetenz (und des Nichtwissens) dagegen ist ein Universum" (Reichenbach 2007: 15). Dementsprechend ist auch die schulische Welt bevölkert von Nichtwisser/ innen, von mathematischen, physikalischen, juristischen, geographischen, geschichtlichen, politischen und vielerlei anderer Art von angeblichen „Analphabet/innen" - und eben auch von finanziellen. Wo ist das Problem? Wozu ausgerechnet Finanzbildung und nicht etwa Rechtsbildung, Lebensführung oder Medizin? Solche Fragen werden sorgfältig vermieden, seriöse Antworten dazu fehlen.

\section{Finanzen und Bildung im Kontext}

Aus Wissenslücken kann man nicht auf Bildungslücken schließen. Schauen wir uns also etwas genauer an, was in der orthodoxen ${ }^{2}$ Wirtschaftsdidaktik unter Finanzbildung verstanden wird. „Finanzielle Allgemeinbildung bezeichnet den Prozess zur Entwicklung von Finanzkompetenz" und Finanzkompetenz ermöglicht Orientierung auf dem Finanzdienstleistungsmarkt, Organisation der privaten Finanzen samt entsprechendem Handeln sowie Beteiligung ,an der Analyse und Gestaltung der institutionellen Rahmenbedingungen des Finanzdienstleistungsbereichs" (Kaminski \& Friebel 2012: 40).

Hier treffen wir auf einen bemerkenswert bescheidenen Bildungsbegriff, der Bildung als einen Prozess begreift, der mit dem Zustand der Kompetenz sein Ziel erreicht hat und dann solange ruht, bis die Praxis nach neuen Kompetenzen ruft. Dieser Ansatz stellt das herkömmliche Bildungsverständnis auf den Kopf; er stuft Bildung zu einem Mittel für Kompetenz herab.

Eine dreifache inhaltliche Reduktion dieser „Bildung" kommt noch hinzu. Erstens werden die Lernenden wissenschaftlich, willkürlich und einseitig,

\footnotetext{
Für Unterscheidungen innerhalb der Fachdidaktik nutzen wir hier die wirtschaftswissenschaftliche Terminologie und nennen diejenigen fachdidaktischen Ansätze „orthodox“, für die mindestens zwei der folgenden fachdidaktischen Charakteristika gelten: Monodisziplinarität, Monoparadigmatizität, normativer Ökonomismus (Zweckrationalität, Rechenhaftigkeit, Effizienz als dominante Erziehungsziele oder Wertmaßstab), asoziales Akteurkonzept (isoliert-autonomer Akteur/in). Das entspricht dem Kern dessen, was in der Volkswirtschaftslehre als „Orthodoxie“ klassifiziert wird.
} 
in einen einzelnen ökonomischen Denkansatz hineingezwungen, die sogenannte „Ökonomik“ nach Karl Homann und Andreas Suchanek (Kaminski \& Friebel 2012, 26-28, 52-56). Diese paradigmatische Einseitigkeit zieht eine Simplifizierung nach sich und verhindert wissenschaftliche Multiperspektivität. Sie stellt einen Verstoß gegen das Neutralitätsgebot staatlicher Bildung im Rahmen der Schulpflicht in dem Sinne dar, dass eine Parteinahme für eine bestimmte wissenschaftliche Position nicht legitim ist. Zweitens beschränkt sich die "finanzielle Allgemeinbildung“ ganz überwiegend auf das Ziel eines funktional angemessenen Agierens im Komplex Finanzdienstleistungen und schränkt Bildungsprozesse damit auf ein Minimum ein. Drittens bleibt die wichtige Frage außen vor, ob die aktuellen Anforderungen an die Menschen, ihr finanzielles Wohl unter den gegenwärtigen ökonomischen Bedingungen und bei auch monetär sehr ungleichen Start- und Verwirklichungschancen aus eigener Kraft zu erreichen, nicht einfach zu hoch und unrealistisch sind (Willis 2018: 83-84).

Aber man kann den Anspruch von Finanzbildung (financial literacy) noch tiefer hängen. Man kann ihn auf „Kenntnisse und Fähigkeiten, Einstellungen und Verhaltensweisen, die es den Menschen ermöglichen, in konkreten finanziellen Situationen eine ökonomisch sinnvolle Entscheidung zu treffen und finanzielle Probleme zu lösen", beschränken, daran messen, „[w]ie gut eine Person ihre finanziellen Angelegenheiten regeln kann" und dazu Indikatoren wie das Wissen über Inflation, Kredit, Zinsen und Zinseszinsen heranziehen (vgl. Greimel-Fuhrmann 2012/13: II-IV). Ein gebildeter Mensch ist dann eine Person, die ihre Alltagsangelegenheiten im Griff hat. Dies bezeichnete man früher als einen kundigen, jüngst einen kompetenten, nicht aber als einen gebildeten Menschen.

Wer angesichts dieser Verflachung, Instrumentalisierung und Einseitigkeit von Bildung ein ernsthaftes Unbehagen empfindet, der muss allerdings auch sagen können, was „echte Bildung" im Themenfeld Finanzen bedeutet. Zunächst muss man betonen, dass Bildung etwas mit der Person zu tun hat; Bildung betrifft, berührt, bestimmt, vertieft, verändert die Persönlichkeit. Bildungsprozesse beziehen sich auf drei Verhältnisse der Person, auf ihr Selbstverhältnis, auf ihre Weltverhältnisse und auf ihre Sozialverhältnisse (Koller 2018).

\subsection{Finanzlernen oder Finanzbildung?}

Was wäre „echte“ Bildung? Eine Finanzbildung, für die die Bezeichnung Bildung nicht nur ein Euphemismus bleibt, konzentriert sich auf diese drei personalen Verhältnisse. Die Schüler/innen bearbeiten dann Fragen, mit denen sie ihr Selbstverhältnis entwickeln, zum Beispiel: Was macht Geld mit mir? Wie will ich, wie soll und wie muss ich mit Geld umgehen? Sie beantworten Fragen, mit denen sie ihre eigene Weltorientierung aufbauen, beispielsweise: Wie funktioniert Geld? Was bewirkt Geld? Was ist Finanzialisierung und was bedeutet sie für die Welt in der ich lebe, und die Welt in der ich leben möchte? Wie wirkt die „Finanzialisierung" auf mein eigenes Leben? Sie setzen sich mit Fragen auseinander, die die eigenen Sozialverhältnisse reflektieren, wie etwa: Was bedeutet Geld für meine Beziehungen zu anderen? Wie prägt Geld das gesellschaftliche Zusammenleben? Wie beeinflusst Geld die Politik? Kann und will ich gesellschaftliche Geldverhältnisse ändern?

Was kann man an der Gegenüberstellung des instrumentalistischen und des personalen Begriffs von Bildung erkennen? Die beiden Bildungsbegriffe bezeichnen die Pole einer wissenschaftlichen, politischen, gesellschaftlichen und bildungspolitischen Auseinandersetzung um Denkweisen und Weltbilder. Wir erleben seit einiger Zeit zahlreiche Vorstöße zur Umprogrammierung des Denkens der Individuen, die auf eine Ökonomisierung der Gesellschaft und der Bildung abzielen (Fohrmann 2016). In den beiden hier interessierenden Konfliktfeldern Stundentafelstruktur und Bildungsphilosophie geht es wesentlich auch um Wirtschaft und um die Hegemonie von Wirtschaftsbildern, Wirtschaftswissenschaften und Wirtschaftspolitik.

Dieser Deutungskampf erstreckt sich auf Bildung im Allgemeinen und auf ökonomische Bildung im Besonderen. Die hier skizzierte reduktionistische Auffassung von Bildung läuft darauf hinaus, Differenzierung durch Nivellierung zu verdrängen, indem man alles Lernen unterschiedslos „Bildung“ nennt. Dies findet man in exemplarischer Verkürzung bei Hans Kaminski, wenn Bildung als „sozialpraktischer Begriff“ vorwiegend auf die Bewältigung konkreter Lebenssituationen bezogen wird (Kaminski 2012: 188). Die Stiftung Warentest drückt sich differenzierter aus als manch ein Protagonist von Finanzbildung, wenn sie „praktisches ökonomisches Wissen rund um Finanzprodukte“ vermitteln sowie „kritisches Konsumverhalten und die finanzielle Entscheidungskompetenz von Schülerinnen und Schülern" fördern will, ohne zu behaupten, dass sie damit Finanzbildung betreibe (Stiftung Warentest 2015). Wenn aber Bilden mehr bedeuten soll als instrumentelle, ökonomistische Vermittlung von Finanzwissen und -können (Finanzausbildung) und Erziehung zum/zur funktionierenden Kunden/in für Finanzdienstleister/innen, einem/r Kunden/in, der/die auch ein bisschen über Regulierung nachdenken darf, solange er/sie die vorgegebenen Denkschemata nicht verlässt, dann braucht man einen anspruchsvolleren Begriff. 
Einen Vorschlag dazu macht Udo Reifner, wenn er verlangt, „[f]inanzielle Allgemeinbildung müsste Konsumenten die Fähigkeit vermitteln, ihre eigenen Bedürfnisse und Ressourcen zu erkennen, sie in Bezug zu den Möglichkeiten der Bedürfnisbefriedung durch marktmäßige Finanzdienstleistungsangebote zu setzen und bei ihnen Verständnis dafür erzeugen, dass sie ihre Rechte, ihre Marktmacht und politischen Einfluss einsetzen müssen, damit Finanzdienstleistungen für sie einen Nutzen bringen" (Reifner 2017: 129). Reifner plädiert für eine „bedürfnisorientierte finanzielle Allgemeinbildung", die Verbraucherorientierung, Verbraucheraufklärung, Verbraucherschutz, Selbstbewusstsein und Kritikfähigkeit in den Mittelpunkt stellen soll (Reifner 2011: 18, 22). Sein Ansatz stellt „Reflexion" als ein zentrales Element personaler Bildung heraus, schließt aber auch praxisorientierte Kompetenzen ein.

Diese Auffassung von Bildung kommt dem näher, was der Philosoph Peter Bieri in seiner Schrift „Wie wäre es, gebildet zu sein?" als Bildung versteht (Bieri 2008: 13). „Bildung ist etwas, das Menschen mit sich und für sich machen: Man bildet sich. Ausbilden können uns andere, bilden kann sich jeder nur selbst. [...] Eine Ausbildung durchlaufen wir mit dem Ziel, etwas zu können. Wenn wir uns dagegen bilden, arbeiten wir daran, etwas zu werden - wir streben danach, auf eine bestimmte Art und Weise in der Welt zu sein."

Sollen junge Menschen sich zu möglichst autonomen Persönlichkeiten entwickeln können, dann brauchen sie Gelegenheiten darüber nachzudenken, was Finanzerziehung mit ihnen machen soll und ob sie diese Veränderung ihrer persönlichen Art und Weise in der Welt zu sein wollen oder nicht. Solche Metareflexionen über die Effekte von Bildungsmaßnahmen, die einer funktionalistischen Verkürzung der Lebensführung vorbauen, werden den Lernenden in den gängigen Ansätzen von „Finanzbildung“ jedoch systematisch vorenthalten.

Wenn Begriffe dem Begreifen dienen und wenn das auch für den Begriff Bildung gelten soll, dann muss man fortan die Finanzausbildung für finanzielle Laien von der Finanzbildung für Personen unterscheiden. Indem sie Bildung auf die persönlichen Selbst-, Welt- und Sozialverhältnisse bezieht, nimmt die Sozioökonomiedidaktik diese Unterscheidung ernst, sie entscheidet sich für Finanzbildung. Dagegen will Finanzerziehung ein vorbestimmtes Denken über Geld und Kapital, Anlegen und Vorsorgen vermitteln und finanzmarktaffine Einstellungen sowie individualistisch-privates Investor/innenhandeln erzeugen. Finanzerziehung ist normativ und paternalistisch, sie geht damit über die funktionalistischinstrumentalistische Finanzausbildung für Laien hinaus.
Bildung braucht Wissen und Können, sonst bleibt sie leer. Für die sozioökonomische Bildung macht eine separate Finanzbildung keinen Sinn. Das Themenfeld Finanzen ist unverzichtbarer und integraler Bestandteil der Teildomäne Wirtschaft in der Gesellschaft, dem Gegenstandsbereich der sozioökonomischen Bildung. Eine Zerlegung in einzelne Bildungen - etwa Finanz-, Unternehmens-, Beschäftigungs-, Außenwirtschafts-, mikro-, makro-, fiskal- oder wirtschaftspolitische Bildung - würde die sachlichen Zusammenhänge willkürlich auseinanderreißen und wäre für den systematischen Erwerb von wirtschaftlichem Wissen und Können kontraproduktiv.

Auf Wirtschaft bezogenes Wissen und Können hier verstanden als sozialwissenschaftliches Wissen und Können zum Gegenstandsbereich Wirtschaft muss sozioökonomiedidaktischen Kriterien genügen, damit man es als bildungsrelevant auszeichnen und in Lehrplänen und Lehr-Lern-Materialien für Schule und Unterricht aufnehmen kann. Es muss an dieser Stelle reichen, die Leitprinzipien sozioökonomischer Bildung kurz in Erinnerung zu rufen, denn sie sind andernorts erläutert (vgl. Fridrich \& HofmannSchneller 2017; Hedtke 2015: 24-28, 2018: 46-65). In der Anwendung müssen die Prinzipien in Form von Kriterien konkretisiert werden. Im Subjektbezug gelten die Prinzipien Subjektorientierung und Bildungsorientierung, im Gegenstandsbezug Problemorientierung und Kontextualisierung, Wissenspluralität und Diversität sowie Kontroversität und Kritik und schließlich im Wissenschaftsbezug Wissenschaftsorientierung, Sozialwissenschaftlichkeit und Multiparadigmatizität.

Sozioökonomiedidaktische Leitlinien und Prinzipien helfen, bei der Konstruktion von Curricula, Lehr-Lern-Materialien und Unterrichtseinheiten, den Blick auf das Wesentliche zu richten. Für Bildungsprozesse zum Gegenstandsbereich Wirtschaft in der Gesellschaft greifen wir Kontextualisierung exemplarisch heraus, sie ist als inhaltlicher Anspruch und unterrichtliches Verfahren mindestens dreifach relevant: für die Analyse, für die Person und für das Curriculum. In der Analyse des Gegenstandsbereichs Wirtschaft geht es darum, die soziale, räumliche, zeitliche und politische Kontextgebundenheit wirtschaftlicher Phänomene mit Hilfe von Ergebnissen der sozialwissenschaftlichen Wirtschaftsforschung aufzudecken und zu erklären. Für die Weltorientierung der sich bildenden Person steht die Thematisierung und Aufarbeitung von Kontextreferenz im Zentrum, weil sie eine Voraussetzung für ein angemessenes Verständnis von Wirtschaft sowie für die eigene Positionierung und Orientierung in den Wirtschaftswelten ist. Für das Gesamtcurriculum eines Bildungsgangs zielt Kontextualisierung auf eine systematische Einbettung 
der sozioökonomischen Bildung in die Domäne der gesellschaftlichen Bildung. Diese schließt selbstverständlich die geographische Bildung ein (Fridrich 2018: 84-89).

Eine angemessene Auseinandersetzung mit dem Finanzkomplex in der sozioökonomischen Bildung verlangt Aufklärung über den Stand der Wissenschaft und über Interessenlagen, Strategien und Politiken. Die sozioökonomische Kontextualisierung ist ein Mittel solcher Aufklärung.

\subsection{Bildung oder Interessenpolitik?}

„Bildung ist der beste Anlegerschutz!“, das versichert der Chief Executive Officer der Wiener Börse AG und propagiert damit wortgleich dieselbe Strategie wie das Deutsche Aktieninstitut (Deutsches Aktieninstitut 2013: 4). „Mehr Finanzbildung und weniger Regulatorik“, so bringt ein Magazin für Anlageberater/innen den Tenor des Österreichischen Sparkassentags 2017 auf den Punkt (Fonds professionell Online 2017). Ähnliche Äußerungen aus der Finanzindustrie und ihren Lobbyverbänden häufen sich. Unter dem Eindruck der Finanzkrise forderte eine deutliche Mehrheit in Wissenschaft und Politik, dass eine „Neuausrichtung auf den Schutz des Verbrauchers an den Finanzmärkten zu unserem obersten wirtschaftlichen Prioritäten gehören“ muss (Akerlof \& Shiller 2009: 210).

Viele Wirtschaftsdidaktiker/innen, Wirtschaftspädagogen/innen tragen mit ihren Forderungen nach Finanzausbildung und individualisierenden Finanzerziehungsprogrammen zur politischen Amnesie und zur Diskreditierung von Regulierung bei. Zu glauben, man könne den „irrationalen Handlungsweisen“ der Akteure und ihren Folgen (Akerlof \& Shiller 2009: 239) durch eine Umprogrammierung der schulischen Lehrpläne beikommen, ist ein naiver und gefährlicher Irrglaube. Kritiker/innen wie Lauren Willis bezeichnen deshalb die vorherrschenden Vorstellungen der sogenannten Finanzbildung als „ein eigenartiges, ja perverses Konzept [...], denn es ist belastet mit dem Glauben, dass der oder die Einzelne sich ändern kann und sollte, um die Bedürfnisse des Marktes zu befriedigen" (Willis 2018: 85).

Man kann dieses finanzmarkt- und bildungspolitische Vorhaben der Finanzerziehung auch als Verhöhnung der Normalbürger/innen bewerten, die den Finanzunternehmen als private, unprofessionelle Gelegenheitsanleger/innen und im Falle der Jugendlichen demnächst als Kunde/in gegenübertreten. Hier manifestiert sich ein Messen mit zweierlei Maß. Auf der einen Seite geht es um ein politisch gewolltes $\mathrm{Zu}$ rückstutzen von Ansprüchen auf finanzielle Absicherung auf Eigenverantwortung und Anlegerbildung sowie um die Verlagerung kollektiver Vorsorge auf das Individualrisiko der einzelnen Bürger/innen. Auf der anderen Seite bedient die Politik die Forderungen global agierender Konzerne nach gesetzlichem, gerichtlichem und zusätzlichem, schiedsgerichtlichen Investitionsschutz, die etwa im Kontext der Verhandlungen über TTIP erhoben wurden und beim CETAAbkommen zwischen der EU und Kanada in Form eines Multilateralen Investitionsgerichtssystems (ICS).

Kritik kommt gelegentlich auch aus der Branche selbst. So kommentiert die Geschäftsführerin des Online-Vermögensverwalters Whitebox, ein Konkurrent der etablierten Fondsindustrie, für die Tageszeitung Die Welt die „scheinheilige Forderung nach mehr Finanzbildung" (online, 8.8.2018). Nüchtern betrachtet ziele Finanzbildung auf die Generierung von weiteren Gewinnen aus Provisionen und Verwaltungsgebühren, indem man mehr Menschen aktiviere und auf den Markt für Finanzprodukte ziehe. Zugleich führt die Finanzindustrie ein Mehr an Finanzwissen gegen unliebsame staatliche Regulationen des Sektors ins Feld, weil sie sicher sein kann, dass die Informations- und Machtasymmetrie zwischen einzelnen Privatkunden/innen und Anlageanbieter/ innen immer so zu ihren Gunsten ausfallen wird, dass sie auch besser finanzinformierte Kunden/innen nicht fürchten muss. Vielmehr hinkt das Anlegerwissen immer hinter der Entwicklung neuer Produkte hinterher, und die „Informationsüberlastung des Verbrauchers stellt im Rahmen der Anlageberatung [...] eher die Regel dar" (Kohlert \& Oehler 2009: 89; Oehler \& Wendt 2017: 182).

Die derzeit propagierte Form der Finanzerziehung nützt (auch) der Finanzindustrie, indem sie Hemmschwellen abbaut, junge Erwachsene mit der falschen Sicherheit schulischen Halbwissens für die Kapitalmärkte motiviert und den Unternehmen so neue Kunden/innen zuführt. Zusammen mit einer Rentenpolitik, die in Deutschland durch Absenken des Rentenniveaus und Propaganda für private Altersvorsorge über die Kapitalmärkte („Riester-Rente“) gekennzeichnet ist, generiert das Provisionen, Profite und CEO-Einkünfte. Diese Politik läuft auf eine Subventionierung der Finanzindustrie hinaus; Tim Engartner resümiert das als "Rendite statt Rente“ (Engartner 2016). Sie schafft Arbeitsplätze im „rasant expandierende[n] Sektor von Finanzbildungseinrichtungen" (Weber 2010: 379).

All das ist weder illegal, noch illegitim, muss aber als ein wichtiger politisch-ökonomischer Wirkmechanismus in der Finanzbildung kontrovers thematisiert werden, wenn die jungen Menschen für ihr Leben relevante Teile des Finanzsystems verstehen sollen. Dazu müssen sie auch diskutieren, ob und in welchem Ausmaß Vertreter/innen der Finanzindustrie einen privilegierten Zugang zu Regierungen, Parlamenten und 
Parteien haben, und, ob diese erfolgreiche Regulierungen in ihrem Sinne bewirken können - insbesondere durch Ausnutzung der „Informationsasymmetrie zwischen Regulierern und Regulierten" (Mayntz 2012: 395).

Zudem kann die Finanzindustrie immer mittels politisch-ideologischem Framing die Öffentlichkeit gegen eine vermeintliche „Bevormundung" der Menschen durch "den Staat" mobilisieren und fordern, dass alle selbst „frei“ entscheiden können und sollen, da sie ja durch die Finanzerziehung kompetent geworden seien. So beklagt der Präsident des Österreichischen Sparkassenverbandes „überzogene“ Schutzbestimmungen, die die Konsumenten/innen „belasten“, sodass sie durch ein „Zuviel an gesetzlichem ,Schutz teilweise wie ,besachwaltete' Menschen behandelt werden" (Fonds professionell Online 2017). Auch das Deutsche Aktieninstitut fordert von der Bundesregierung „Ökonomische Bildung statt Paternalismus in der Anlageberatung“, Regulierung dürfe „den Anleger nicht entmündigen" (Deutsches Aktieninstitut 2013: 8). Die „Besachwaltung“ der Menschen, die ihre Kunden/innen sind, hat die Branche am liebsten selbst in der Hand.

Auch im Vorfeld bildungspolitischer Entscheidungen zur Ausweitung ökonomischer Themen im Schulunterricht bis hin zur Einrichtung eines Separatfachs Wirtschaft kann man meist Einflussnahmen von Konzernen, Wirtschaftsverbänden, Wirtschaftskammern, Unternehmensstiftungen und unternehmensnahen Think Tanks beobachten; die Wirtschaftspresse wirkt dabei unterstützend mit (Wendt et al. 2016). So können monetäre Ressourcen in öffentlich-mediale Aufmerksamkeit und politisches Agenda-Setting konvertiert werden, auch wenn man in pluralen Gesellschaften mit Pressefreiheit beides nicht zentral steuern kann. Fakt ist jedoch, dass kritische Berichterstattung über Finanzerziehung sehr selten vorkommt, während die uneingeschränkt positive mediale Resonanz dominiert und damit zum einseitigen Framing beiträgt (Weber 2010: 380).

\subsection{Willkür oder Wissenschaft?}

Wie steht es um die wissenschaftlichen Grundlagen für die Forderung nach mehr Finanzwissen durch Schulunterricht? Wer Finanzausbildung für Laien anstelle eines vernünftigen, sprich, kundenfreundlichen Designs der Institutionen des Finanzmarkts fordert, der offenbart Defizite in seinem wirtschaftswissenschaftlichen und wirtschaftspsychologischen Wissen. Denn er stellt die Veränderung der Individuen in den Mittelpunkt seiner Bemühungen, nicht die Änderung der Institutionen (Willis 2018: 81-82). Wirtschaftswissenschaftler/innen setzen bekanntlich mehrheitlich weniger auf Information und kaum auf Umerziehung des/der Einzelnen, sondern eher auf kollektiv geltende Regeln und Institutionen, auf gezielte, mehr oder weniger automatisch wirkende Anreize und Sanktionen. Viele Wirtschaftswissenschaftler/innen halten „Preise und andere Marktinstrumente" für die Mechanismen, die die Wünsche der Akteure beschränken und ihre Tauschaktivitäten koordinieren (Becker 1993: 4). Gebhard Kirchgässner bringt die programmatische Perspektive auf den Punkt: „Die Ökonomie ist [...] eine Wissenschaft von der Veränderung der Verhältnisse. Menschliches Verhalten kann dadurch beeinflusst bzw. verändert werden, dass die Verhältnisse, unter denen die Menschen agieren [...] verändert werden" (Kirchgässner 2008: 26).

Was heißt das für die Auseinandersetzung mit dem Themenkomplex Finanzen? Wenn sie Bildungswirkung entfalten soll, dann kommt es hier auf eine demokratische Debatte über die Veränderung (und Veränderbarkeit) der Finanzinfrastruktur an, über die Vermeidung von Unsicherheit, die Verteilung von Risiken, die Pufferung von großen Schwankungen, und die Bekämpfung von Armut und Ungleichheit (Willis 2018: 86). Dafür braucht man, so Lauren Willis, finanzinformierte Bürger/innen, ein bürgerbestimmtes Finanzsystem, Bürger/innen, die politisch urteilen und entscheiden können, nicht aber privatisierende und voneinander isolierte Investor/innen (vgl. Retzmann \& Seeber 2016: 21). Willis' Unterscheidung von Bürger/in und Investor/in entspricht in etwa der einschlägigen Gegenüberstellung von Citoyen und Bourgeois.

Auch Vertreter/innen der Wirtschaftspsychologie und der Verhaltensökonomik erwarten wenig Wirkung vom Wissen auf das Handeln von Individuen. Das belegt beispielsweise der Verhaltensökonom Dan Ariely in einem Buch mit dem programmatisch skeptischen Titel „Denken nützt zwar, hilft aber wenig. Warum wir immer wieder unvernünftige Entscheidungen treffen" (Ariely 2008). Die Unvernunft betrifft insbesondere den Umgang mit Geld, Investitionsund Finanzentscheidungen, etwa die „Willkürlichkeit und Unstetigkeit des Sparverhaltens" oder die erhebliche Abhängigkeit der Investitionsentscheidungen von "psychologischer Befindlichkeit" (Akerlof und Shiller 2009, 169-222, zit. 187, 204). Schließlich kann man mit Finanzbildung für Jugendliche eher wenig gegen die individuell unkontrollierbaren animal spirits, die unüberwindbare Zukunftsungewissheit, die extreme Komplexität der den Privatanleger/innen zugänglichen Finanzmarktprodukte und die dementsprechend heillose Überforderung ausrichten (Remmele 2016).

Generell konstruieren auch die Anbieter/innen selbst Komplexität, wenn dies ihre Position gegenüber den Privatkunden/innen (sowie der Politik und der Finanzaufsicht) stärkt, d.h. im konkreten Fall die 
Informations- und Machtasymmetrie zugunsten der Finanzkonzerne sichert (Dullien 2013). Man muss schon sehr sorgfältig analysieren, wo schulisches Lernen in diesem Feld wahrnehmbare und wichtige Unterschiede im Anlegerhandeln hervorbringen kann und wo nicht. Generell scheint die empirische Forschung zu zeigen, dass Finanzbildung wenig Wirkung im Handeln erzeugt und dass auch die Bewusstmachung unbewusster Prozesse nichts bewirkt (Aprea et al. 2016; Willis 2018: 83-84).

Den Protagonisten/innen der Finanzerziehung käme also eine Weiterbildung in moderner Volkswirtschaftslehre und Verhaltensökonomik zugute, um willkürliche durch wissenschaftliche Argumentationen ersetzen zu können. Aber es verlangt sehr viel politisch-ökonomische Naivität zu glauben, dass neues Wissenschaftswissen wirken würde und die Chefetagen der Finanzindustrie zur Abkehr vom Projekt Finanzerziehung brächte. Viel eher sind es die Interessen der Vorstände, Unternehmen und Branche, die ihre Bildungsaktivitäten antreiben. Ihre Forderungen nach „Finanzbildung" fallen also vor allem in die Kategorie Lobbyismus.

\subsection{Privatsache oder Politikum?}

Finanzindustrielle Lobbyarbeit richtet sich aber nur nachrangig auf Schule, im Zentrum steht die Einflussnahme auf die Finanzmarktregulierung. Die öffentliche bildungspolitische Debatte um die „Finanzbildung" eignet sich deshalb bestens, um vom politischen Kernproblem einer angemessenen Regulierung der Finanzindustrie und deren Durchsetzung abzulenken. Dagegen gehört die Auseinandersetzung mit den institutionellen und politischen Rahmenbedingungen wirtschaftlicher Phänomene und Probleme zu den Kernthemen sozioökonomischer Bildung. Die Regulierung der Finanzmärkte wie zum Beispiel die Verteilung der Anlagerisiken zwischen Wertpapieremittenten, Vermittler/innen, Berater/innen und Verkäufer/innen sowie privaten Anleger/innen (Risikomanagement), die Besteuerung von Kapitalerträgen oder die Politik in Finanzkrisen wirken unmittelbar oder mittelbar auf private, individuelle Finanzentscheidungen ein. Sie müssen deshalb integraler Bestandteil von Finanzbildung sein (Willis 2018: 87).

Wer der Öffentlichkeit weismachen will, Finanzbildung für jedermann sei ein probates Mittel gegen zukünftige Finanzkrisen, verdrängt die Tatsache, dass es die Elite des Finanzwesens und die Spitze des Finanzwissens waren, deren systemisch-politische und unternehmerische Entscheidungen sowie Lobbyismuserfolge die letzte Finanzkrise wesentlich verursacht haben (Weber 2010: 389-392). Das Finanzwissen der Finanzeliten zeitigte hier katastrophale Folgen für die
Staatshaushalte und für die breite Masse, nicht jedoch für die Spitzengruppe unter den Eigentümer/innen von Kapitalvermögen und kaum für die Finanzindustrie. Es kommt offensichtlich darauf an, welches Wissen man in wessen Interesse verwendet und verbreitet, und wer die Macht hat, die Finanzwelt nach seinem Wissen zu gestalten. Das war in den Jahrzehnten vor der Krise vor allem die Finanzbranche, die von der Politik mächtig gemacht wurde (Young 2014: 69-70). Heute verhindert insbesondere „die Abhängigkeit der Politik vom Finanzsystem" eine angemessene Regulierung der Finanzmärkte, die sich mehrfach manifestiert mit Blick auf Arbeitsplätze, Steuereinnahmen, Wirtschaftswachstum und kreditförmiger Staatsfinanzierung; das Finanzsystem gilt deshalb als „politisch nicht mehr steuerbar" und ,interventionsresistent" (Mayntz 2014: 12-14). Das betrifft in erheblichem Maße auch die vielfältigen kriminellen, illegalen, halblegalen und illegitimen Aktivitäten der Finanzindustrie, etwa in den Bereichen Steuerminimierung, Steuerhinterziehung, organisierter Kriminalität, Korruption, Geldwäsche oder Risikoabwälzung auf die Steuerzahler/innen (z.B. Masciandaro 2004; Platt 2015).

Man sieht, Finanzwirtschaft und Politik lassen sich nicht trennen. Im Hintergrund stehen hier grundlegende und kontroverse Vorstellungen von Staat und Staatsaufgaben, vom Verhältnis von Staat und Privatwirtschaft, von wirtschaftspolitischer Regulierung und Deregulierung. Die Kontroversen betreffen auch grundsätzlich unterschiedliche Konzeptionen von Rentensystemen, privater Anlegerkultur und kapitalmarktgedeckter Altersvorsorge, etwa in Deutschland und Österreich (z. B. Blank et al. 2016).

Findet Finanzbildung vor diesem Hintergrund ohne politische Kontextualisierung und ohne bürgerschaftliche Diskussion der Frage statt, wie eine Gesellschaft ihren Finanzsektor gestalten soll und kann, dann hat sie die Bezeichnung Bildung nicht verdient. Denn sie schließt die Reflexion und Weiterentwicklung der Weltverhältnisse der Lernenden aus dem Bildungsprozess aus. Sie bleibt funktionalistische Finanzausbildung. Dabei liegen wichtige Kontroversen auf der Hand:

Soll der Staat als Sachwalter eines Gemeinwohls, als Advokat der Versicherten und Kleinanleger/innen agieren oder als Förderer von Finanzindustrie und Großinvestor/innen? Welche Interessengruppen und -koalitionen sollen politisch das Sagen haben? Was legitimiert den Staat dazu, in einer liberalen Demokratie mit relativ freien Märkten seine jungen Bürger/ innen zu einer ganz bestimmten Finanzkultur zu erziehen? Ist es demokratisch legitim, die von der nichtdemokratischen OECD lancierte politische Strategie der Förderung von Anlegermentalität und Rentabili- 
tät als Eigenwert und die Politik der Umerziehung in Richtung financial literacy ohne Partizipation und politische Debatten umzusetzen? Soll man statt der Bürgergesellschaft eine Eigentümergesellschaft formieren, als deren Leitfiguren Kapitalisten/innen, Investor/ innen und Rentiers firmieren (Deutschmann 2013)? Kann und soll man die Macht der Finanzindustrie begrenzen? Was ist das richtige Politikkonzept? Individualisierung, Privatisierung, Kommerzialisierung, Vermarktlichung von finanziellen Chancen und Risiken? Oder ist eine kollektive, solidarische, demokratische Risikoabsicherung die bessere Variante? Ist ein „wirtschafts-, verteilungs- und handelspolitisch aktiv eingreifender Staat [...] die unerlässliche Voraussetzung dafür, um Kapitalismus und eine faire Verteilung von Einkommen und Vermögen miteinander vereinbar zu machen" (Deutsches Aktieninstitut 2013: 66)? Oder sollen sich Gesellschaft, Demokratie und Politik damit abfinden, dass das Matthäus-Prinzip ein exponentielles Wachstum der Vermögensungleichheit bewirkt (Kranzinger 2016: 135-141)?

Die orthodoxe Wirtschaftsdidaktik ignoriert mehrheitlich die letztgenannten Fragen. Sie verengt beispielsweise die Auseinandersetzung mit dem Themenkomplex Altersvorsorge stark und entpolitisiert sie so. Funktionalistische Finanzausbildung setzt den Jugendlichen die derzeitigen finanziellen Verhältnisse, Verteilungsstrukturen und sozialpolitischen Rahmenbedingungen als gegeben vor. Das entmündigt sie als Bürger/innen. Es läuft faktisch darauf hinaus, dass sie sich damit abfinden, darin zurechtfinden und darauf konzentrieren sollen, für die private Altersvorsorge am Kapitalmarkt mehr oder weniger passende Finanzprodukte zu identifizieren, zu kaufen und zu verwalten.

Im Ergebnis werden sie auch daran gehindert, sich grundsätzlich mit der Frage auseinanderzusetzen, ob der politisch, medial und unterrichtlich erzeugte Zwang zum individuellen, laienhaften, fortlaufenden Beobachten, Entscheiden, Handeln auf Kapitalmärkten samt der endlosen Sorge um die Vorsorge wirklich ihren persönlichen Vorstellungen vom guten Leben entspricht. Ihnen wird die Möglichkeit vorenthalten, sich mit Alternativen der Altersvorsorge auseinanderzusetzen und gegebenenfalls dafür politisch aktiv zu werden. Dagegen konfrontiert eine kritische Kontextualisierung, die reines Anpassungslernen überwindet und auf Bildung und Persönlichkeitsentwicklung zielt, die Jugendlichen auch bei finanziellen Angelegenheiten mit der Grundfrage „Wie wollen wir leben?".

Konkret bedeutet das beispielsweise, dass man die Frage diskutiert, ob es besser wäre, kollektive Institutionen kapitalmarktorientierter Vorsorge zu konstruieren, womöglich in Form staatlicher oder öffentlich-rechtlicher Organisationen, auf die sich das Individuum verlassen kann, obwohl es sich kaum da- rum kümmern muss (Corneo 2018) - ironischerweise war und ist das beim herkömmlichen staatlichen Rentensystem weitgehend der Fall (tu felix Austria?). Angesichts der ständig wachsenden Komplexität des Alltagslebens und der zunehmenden Zeitknappheit muss die institutionelle Entlastung des Individuums die zentrale Leitidee sein, nicht das Aufbürden vieler weiterer Entscheidungen in einer Gesellschaft, die bereits immer mehr Bereiche entscheidungsförmig organisiert und dort einen Entscheidungszwang institutionalisiert (Schimank 2006). In die Richtung „to simplify people's lives" gehen auch einige wenige Politikvorschläge der OECD (OECD 2016: 60). Mehr an den Bedürfnissen der Personen, als an den Interessen der Finanzindustrie, ausgerichtete Institutionen würden auch „faule Anleger/innen“, „gehetzte Multitasker/innen“" oder ,eilige Konsument/innen“ als Zielgruppen bedienen, statt mit massivem Mitteleinsatz vergeblich zu versuchen sie zu Miniaturinvestor/innen umzuerziehen.

Diese Debatte muss man nicht neu erfinden. So haben beispielsweise Thorsten Hippe und Reinhold Hedtke schon vor der Finanzkrise einen pluralen Ansatz zur Behandlung kapitalgedeckter Rentensysteme im Unterricht vorgestellt (Hippe \& Hedtke 2006). Zu den drei dort behandelten Ansätzen zählt auch ein Modell von öffentlichen monopolistischen Fonds (Hippe \& Hedtke 2006: 38-42). Es schließt zwar die individuelle Wahlfreiheit aus, berücksichtigt aber die verbreiteten Wertvorstellungen Sicherheit, Solidarität und Fairness, belästigt die Menschen nicht mit permanentem Entscheidungsdruck, beruhigt die börsentägliche Sorge um die Entwicklung der Kapitalmärkte, und bietet gegenüber privaten Lösungen Vorteile bei Effizienz, Risikoreduktion und Verwaltungskosten. Das Modell beeinträchtigt allerdings die Gewinnchancen der Finanzindustrie, wenn man davon ausgeht, dass professionelle Fondsverwalter in Rahmen eines einseitigen Monopols günstigere Anlagekonditionen durchsetzen können.

Im Sinne der Prinzipien „Wissenschaftsorientierung" und „Kontroversität" kann es kein Bildungsziel sein, dieses Modell oder ähnliche zu propagieren. Aber es demonstriert, wie man die Monoperspektivität der vorherrschenden Finanzbildung öffnen und die Enge der Investor/innensicht überwinden kann, um als Bürger/in die politisch-ökonomische Problemlage aufgreifen und grundsätzliche Alternativen prüfen zu können (Orientierungswissen; vgl. für Deutschland Nullmeier 2015).

Eine andere Alternative wäre die Umorientierung auf eine Lebenslagenpolitik, die nicht nur die drei Säulen der privaten, öffentlichen und betrieblichen Altersvorsorge berücksichtigt, sondern auch Politiken für Wohneigentum, Vermögenseinkommen und kos- 
tenfreien Zugang zu öffentlichen Gütern entwickelt und durchsetzt, etwa bei Kultur und Verkehr (Nullmeier 2015: 200). Alternativen zur derzeitigen deutschen Situation wären beispielsweise ein Staatsfonds, der in ausländische Aktien investiert und seine Nettoerträge als soziale Dividende an alle Bürger/innen ausschüttet, das ähnliche Modell einer „Deutschlandrente" oder die österreichische Variante der öffentlichen, nach wie vor umlagefinanzierten Rentenversicherung (Blank et al. 2016; Corneo 2018; Knabe und Weimann 2017). Ein deutsch-österreichischer Vergleich läge auch deshalb nahe, weil die beiden Nachbarländer zum selben Typ des Wohlfahrtsstaates gehören, wenngleich das Konkordanzprinzip Österreich von Deutschland auch unterscheidet (Haller et al. 2015: 9). Die drei genannten Vorschläge haben den Vorteil, dass sie die privaten Anleger/innen angesichts der extremen Komplexität der Finanzprodukte entlasten und Entscheidungen dem professionellen Fondsmanagement überlassen. Das schützt allerdings nicht vor Fehlentscheidungen, Opportunismus und Kriminalität vonseiten der Professionellen.

\section{Wirtschaft und Bildung als Konfliktfeld}

Die bisherige Argumentation könnte den Eindruck erwecken, dass es im Feld der Finanzerziehung besonders problematisch zugehe, es sich hier also um spezielle Fälle handele, die man nicht verallgemeinern kann. Aber politisch-ideologisches Framing prägt auch die Inhalte orthodoxer ökonomischer Bildung, die an Schulen etabliert ist. Ein Exempel aus einem Schulbuch illustriert das.

\subsection{Das Verhalten oder die Verhältnisse ändern?}

Nehmen wir das Schulbuch „Praxis Wirtschaft Gesamtband", das der Westermann Verlag für das mittlere Lernniveau und die Fächer Arbeitslehre und Wirtschaftslehre in Realschule, Oberschule und Integrierter Gesamtschule im Bundesland Niedersachsen anbietet (Kaminski 2010). Im Kapitel „Veränderungen in der Arbeitswelt" findet man einen Autorentext zum Themenkomplex „Lebenslanges Lernen“. Dort heißt es: „Gerade jüngere Arbeitnehmer müssen sich mit unterschiedlichen Anforderungen auseinandersetzen. Häufig müssen sie sich nicht nur an die Neuen Technologien, sondern auch an die neuen Gegebenheiten des Arbeitsmarktes anpassen, was z. B. heißt, dass sie nur Teilzeitbeschäftigung ausüben können, befristete Arbeitsverträge haben oder Zeitarbeit ausüben. Auch Selbstständigkeit und Selbstverantwortung wird in den zukünftigen Arbeitnehmern immer mehr abverlangt. Um mit diesen Gegebenheiten zu- rechtzukommen, müsst ihr euch darauf einstellen, in eurer beruflichen Laufbahn flexibel zu sein und dürft nicht darauf hoffen, den einmal gelernten Beruf ohne Veränderung bis zur Rente ausüben zu können" (Kaminski 2010: 259). Zwei Seiten vorher betont der Autorentext, man „muss sich von der Vorstellung trennen, dass ein einmal erlernter Beruf oder eine Ausbildung für das ganze Berufsleben den Arbeitsplatz sichert. Es wird immer wieder notwendig sein, sich beruflich neu zu orientieren, weil die technische Entwicklung den bisherigen Arbeitsplatz überflüssig gemacht hat" (Kaminski 2010: 257).

Die Lernenden werden wiederholt mit Alternativlosigkeit und der Forderung nach individueller Anpassung und apolitischem Denken konfrontiert. Ihre eigenen Arbeitsinteressen und das Bildungsziel einer (möglichst) autonomen Persönlichkeit spielen keine Rolle. Verlangt wird eine bedingungslose Akzeptanz der herrschenden ökonomischen Verhältnisse, insbesondere der Asymmetrie am Arbeitsmarkt und der Herrschaftsverhältnisse im Betrieb. Selbst wenn man die Betrachtung auf eine orthodoxe mikroökonomische Analyse einschränkt, muss man kritisieren, dass das Konzept „Markt“, das Wechselwirkungen zwischen Anbieter/innen und Nachfrager/innen und Anpassungen auf beiden Marktseiten berücksichtigt, durch das Konzept der einseitigen Anpassung auf der Arbeitskraftanbieterseite ersetzt wird. Die Ursachen dafür werden naturalisiert und hier konkret den Technologien und dem Arbeitsmarkt zugeschrieben, Entscheider/in und Akteur/innen - und damit auch: Verantwortliche und Ansatzpunkte für Interessenpolitik - existieren nicht. Die Interessen der Arbeitskraftnachfrager/innen werden ebenso wie Interessenkonflikte zwischen Kapital und Arbeit verdeckt, Möglichkeiten kollektiven und politischen Handelns fehlen, der Staat hat keine Mittel und spielt keine Rolle, nur der/die Einzelne kann und muss sich individuell anpassen.

Diese Naturalisierung der Märkte beherrscht auch die Debatte um das Finanzsystem und die tägliche Berichterstattung über das Börsengeschehen in den Medien, in der die Finanzmärkte als „unpersönliches, abstraktes Kollektiv erscheinen, [... als] ein System [,] das einem gegenübertritt wie die Natur" (Mayntz 2014: 14). Das ist prototypisches marktfundamentalistisches Framing, das systematisch verschweigt, dass die Verhältnisse, in die die Jugendlichen sich gestellt sehen, Ergebnis politischer Programme und Entscheidungen sind (vgl. Willis 2018: 86). Der Marktfundamentalismus, den viele auch als Neoliberalismus bezeichnen, lässt sich für die Zwecke unserer Analyse durch ein naturalistisches Marktverständnis, die radikale Freiheit für Märkte und Finanzmärkte, ein harmonisches Gleichgewichtsdenken, die dualistische 
Gegenüberstellung von Markt und Staat und eine Politik der Entfesselung der Marktkräfte charakterisieren (Ötsch et al. 2018: 14; Young 2014: 67). Marktfundamentalistisches Framing arbeitet darauf hin, die „Brücke zwischen dem Persönlichen und dem Politischen" zu vernebeln (Willis 2018: 88). Insbesondere die „konzeptionelle ,Naturalisierung “ der Märkte und des Finanzsystems „verschleiert handfeste Machtbeziehungen und dient zugleich als Entschuldigung für selbstverschuldete politische Ohnmacht" (Mayntz 2014: 14).

Finanzerziehung und Arbeitsmarkt sind Beispiele für selektive sprachliche Erfahrungen, die durch offizielle Lehrpläne oder staatlich akkreditierte Schulbücher performiert sowie besonders legitimiert und aufgewertet werden. Solche Erfahrungen und deren Wiederholung bestimmen „die für uns sinngebenden Frames“, und so werden „diese Zusammenhänge Teil unseres ganz alltäglichen, unbewussten Denkens, unseres Common Sense" (Wehling 2016: 59). Prinzipiell verhindert selektives Framing zusammen mit der vorsätzlichen Vorenthaltung verschiedener, auch konträrer Frames, „einen bestimmtem Sachverhalt umfassend [zu] denken, ihn, von allen Seiten [zu] beleuchten“" und gefährdet damit die „moralische und ideologische Pluralität in einer Demokratie“ (Wehling 2016: 60). Das gilt insbesondere für die vorherrschende funktionalistische Finanzausbildung, die auf marktfundamentalistischen Denkfiguren fußt und auf neoliberale Umerziehung zielt (Arthur 2012).

Lauren Willis fasst die politische Dimension von Finanzausbildung und Finanzerziehung zusammen: „Finanzbildung als Allheilmittel ist also nicht nur an sich unglaubwürdig, sondern auch eine wahrscheinlich unwirksame politische Maßnahme im Umgang mit den Missständen, auf die sie gerichtet ist. Außerdem schreibt sie die marktfundamentalistischen Mythen fort, dass der Markt sakrosankt ist, der/die Einzelne unausweichlich für sein/ihr finanzielles Unglück verantwortlich ist und dass die Ressourcenverteilung der aktuellen Wirtschaftsordnung nur durch das Handeln Einzelner und nicht durch politischen Wandel verändert werden kann" (Willis 2018: 82). Das kann man gut am Themenkomplex der finanziellen Ungleichheit verdeutlichen, insbesondere an der Ungleichverteilung von Kapitaleigentum, Kapitaleinkünften und Erbmassen.

\subsection{Unwissen über Ungleichheit?}

Neben der Verbreitung marktfundamentalistischer Vorstellungen und Politikmuster erfüllt die orthodoxe Finanzerziehung auch eine gesellschafts-, sozial- und verteilungspolitische Aufgabe: sie verschweigt systematisch die große und wachsende finanzielle Un- gleichheit. Für eine Bildung, die die Bezeichnung verdient, ist das ein inakzeptables Defizit.

Wir greifen zunächst ein Beispiel aus einem Beitrag der Wirtschaftspädagogik zur Messung von financial literacy auf, das sich auf das einschlägige OECDKonzept bezieht (Greimel-Fuhrmann 2012/13, III; vgl. aktuell OECD 2016: 19-21). Das OECD-Set zu finanziellem Wissen enthält offene Fragen und Multiple-Choice-Aufgaben zu Inflation und Geldwertentwicklung, Zins- und Zinseszinsrechnung. Bemerkenswert ist das wenig realitätsnahe Konstrukt von fünf Brüdern, die zu gleichen Teilen ein Geldgeschenk erhalten, aber ein Jahr darauf warten müssen. Die Probanden sollen erkennen, dass das Geld dann wegen der Inflation weniger wert ist und in Abhängigkeit von der vorgegebenen Inflationsrate den richtigen Wert ankreuzen. Die vierte Antwortoption neben "more“, „same amount" und "less" lautet „It depends on the types of things that they want to buy." Wegen der nahezu unbegrenzten Vielzahl an Geldverwendungsmöglichkeiten wäre diese Alternative sachlich passend, aber aus testtechnischen Gründen fehlt sie bei den Antwortvorgaben. Wenn die Befragten sie von sich aus nennen, sollen die Interviewer/innen sie jedoch als richtig werten (OECD 2015: 28-29).

Insgesamt bleibt Finanzwissen auf technischfunktionales, überwiegend mathematisches Wissen beschränkt und gänzlich ohne Bezug zu Gesellschaft und Politik und relevante Problemlagen wie finanzielle Ungleichheit, budgetäre Unsicherheit und persistente Armut. Das ideologisch-politische Framing reicht bis in die einzelne Aufgabenstellung: Die Brüder erhalten gleiche Anteile. Für im OECD-Sinne finanziell Gebildete sollen offensichtlich weder unterschiedliche Anfangsausstattungen, noch unterschiedliche Vermögen und Anlagemöglichkeiten für Ärmere und Reiche, noch unterschiedliche Warenkörbe existieren.

Derartige Finanzbildungskonzepte ignorieren meist die Gruppen, die keine oder wenig Einkünfte haben, deren Einkünfte und Ausgaben heftig und unvorhersehbar schwanken, die mit prekären Beschäftigungsverhältnissen und ungewissen Lebensverläufen konfrontiert sind und für die fast alle der propagierten, individuellen finanziellen Aktivitäten nicht in Frage kommen (Willis 2018: 84). So zählt in Deutschland etwa ein Achtel der Erwerbstätigen zum „zeitstabilen Prekariat" (Allmendinger et al. 2018). Auch in Österreich existiert ein verfestigter Niedriglohnsektor (Geisberger 2017). Für Deutschland beobachtet man einen Trend zur „zunehmenden Verfestigung von Armut“ mit „intergenerationaler Transmission“ (Groh-Samberg 2014). Deutschland besetzt im EU-Vergleich mit einer Niedriglohnquote von 22,5 Prozent den siebtschlechtesten Platz und befindet sich auf Augenhöhe mit Polen, Kroatien, Estland und Griechenland. Ös- 
terreich rangierte mit 14,8 Prozent im oberen Mittelfeld, benachbart von Ungarn, Malta, Spanien und Portugal (Geisberger 2017: 926-928; Daten aus 2014).

Vor diesem Hintergrund hilft Finanzausbildung kaum bis gar nicht. Es käme vielmehr auf arbeitspolitische, arbeitsrechtliche und tarifvertragliche Maßnahmen zur Verstetigung der Beschäftigungs- und Einkommensverhältnisse an, also auf Politik, Auseinandersetzung mit und Mobilisierung für politische Maßnahmen. Die Lernenden müssen sich also mit den kollektiven finanziellen Verhältnissen und ihren individuellen Folgen beschäftigen und lernen, wie sie ihre Verwirklichungschancen auf politischem Wege strukturell verbessern können.

Stellt man all dies in Rechnung, braucht man nicht zuletzt auch realitätsnahe Aufgaben zur Testung der financial literacy. Ein Beispiel könnte vielleicht wie folgt aussehen (betrifft aber wieder nur den in stabilen finanziellen Verhältnissen lebenden Teil der Bevölkerung). Basis für das Beispiel bilden realistische Daten für Deutschland: das Medianeinkommen von Männern aus dem Mikrozensus 2015, eine angenommene Sparquote von optimistischen 5 Prozent, die für die meisten Haushalte unrealistisch hoch ist (2013 lag sie für die beiden mittleren Einkommensdezile zwischen 2,9 Prozent und 3,6 Prozent).

„Matthes erbt $100.000 €$. Matthes lässt sein Geld auf der Bank. Franz erbt $1.000 €$. Auch er lässt das Geld auf der Bank. Franz will auch ein Geldvermögen von $100.000 €$ haben. Als Monatsgehalt bekommt Franz $1.982 €$ ausbezahlt. Davon spart er ab sofort jeden Monat $100 €$. Schätze, wie viele Jahre Franz ungefähr sparen muss, bis er dasselbe Geldvermögen besitzt wie Matthes heute: 20 Jahre, 40 Jahre, 60 Jahre, 80 Jahre oder 100 Jahre?“. Das wäre die einfachste Aufgabenvariante ohne Zins und ohne Zinseszins, die man in komplexeren Varianten einführen muss.

Das wären wirklichkeitsnahe Situationen, an denen die Lernenden erfahren können, was unterschiedliche Anfangsausstattungen im echten Leben bedeuten. Denn neben hohen Einkommen haben „vor allem Erbschaften und Schenkungen eine besondere Bedeutung für die Vermögensakkumulation" (Grabka 2014: 304). In den Varianten mit Zins und Zinseszins illustriert das Beispiel auch den empirischen Befund, dass Kapitalerträge „einen überproportional hohen Beitrag zur Ungleichheit" leisten (Stein 2014: 317). Lässt man das Beispiel mit Zinseszins rechnen, hat man einen konkreten Anlass, über die These von Lauren Willis zur Finanzbildung zu diskutieren, „Der Mythos der Vermögensbildung [...] ist eine Finte" (Willis 2018: 84). Auf jeden Fall gehört eine Auseinandersetzung mit Leistungsprinzip, Meritokratie und Gerechtigkeit zum Kern einer „Finanzbildung“ im sozioökonomischen Sinne.
Aber mehrheitlich blendet die Finanzerziehung die finanzielle Realität in unseren Gesellschaften aus. Sie ignoriert insbesondere die hohe und wachsende finanzielle Ungleichheit. So beschränkt sich beispielsweise die 126-seitige Unterrichtseinheit „Finanzielle Allgemeinbildung" des Handelsblatts und des Oldenburger Instituts für ökonomische Bildung auf die Bemerkung, die aus einer Veröffentlichung „Wirtschaft in die Schule!“ des Deutschen Aktieninstituts stammt: „Damit macht Vermögen den Eigentümer unabhängiger von seinem laufenden Einkommen. Dies ist besonders wichtig für Bezieher abhängiger Einkommen. [...] An dieser Stelle ist aber zu berücksichtigen, dass Vermögen in Deutschland sehr ungleich verteilt sind" (Koch und Friebel 2015: 108). Ergänzend bietet die Unterrichtseinheit eine Grafik zur Vermögensverteilung und eine Aufgabe dazu. Damit ist das Thema abgehandelt.

Das ist kein Einzelfall. Finanzielle Ungleichheit, finanzielle Verwirklichungschancen und finanzielle Umverteilung sind Tabuthemen der Finanzausbildung. Am Beispiel der Vermögensverteilung - und damit am Beispiel des arbeitslosen Einkommens aus Vermögen - zeigt sich die Ungleichheit besonders deutlich. Für Österreich schätzt eine jüngere Studie auf Basis von Daten der Europäischen Zentralbank, dass die Top-1-Prozent der Bevölkerung 41 Prozent des Nettogesamtvermögens besitzen, die unteren 50 Prozent etwa 2,5 Prozent (Ferschli et al. 2017: 26-28, Daten aus 2014-2015). Für Deutschland ergeben Schätzungen einen Anteil von etwa 34 Prozent für die Top-1-Prozent, mehr als ein Fünftel aller Erwachsenen hat kein Vermögen, 7 Prozent sind verschuldet (Westermeier 2015: 131; Grabka 2014: 156, Daten aus 2012). Im europäischen Vergleich haben Deutschland und Österreich eine besonders stark ausgeprägte Vermögensungleichheit (Fessler 2017: 48-52, gemessen am Top-5-Prozent-Anteil).

Dass die Steuerpolitik der beiden letzten Jahrzehnte in Deutschland im Wesentlichen den „Haushalten an der Spitze der Einkommensverteilung genützt hat", bleibt ebenso im Verborgenen wie die politische Option, die Ungleichheit der Markteinkommen und damit auch der Vermögensbildungschancen zu reduzieren (Stein 2014: 320-321). Aber hier herrscht hartnäckige Politikverweigerung, denn im internationalen Vergleich sind die vermögensbezogenen Steuern in Deutschland recht niedrig (Grabka 2014: 304). Strukturelle, kollektive und politische Ursachen der finanziellen Ungleichheit kommen nicht zur Sprache (vgl. Mönter et al. 2016), tendenziell macht die Finanzausbildung den/die Einzelne(n) für sein/ihr Finanzschicksal verantwortlich.

Das ist kein Zufall. Die Entpolitisierung und Individualisierung der finanziellen Verhältnisse, die Abwälzung von Risiken auf die privaten Haushalte 
und die Ausblendung von politischen Alternativen entlastet auch die Politik (Mertens \& Meyer-Eppler 2014: 264-265). Tatsächlich aber legen Demokratien und ihre Regierungen prinzipiell politisch fest, welches $\mathrm{Maß}$ an finanzieller Ungleichheit und damit verbundener Chancenungleichheit sie als erwünscht betrachten und durchsetzen wollen. Würde die Finanzbildung die Ungleichheitsorientierungen in der Bevölkerung und damit auch bei den Lernenden aufgreifen, müsste sie zum einen den Kontroversen zwischen den drei Einstellungstypen Egalitäre, Meritokraten und Liberale Raum geben, und zum anderen die Position erörtern lassen, dass viele in Deutschland und Österreich dem Staat „eine bedeutsame Rolle zur Reduktion von Ungleichheit“ zusprechen (Haller et al. 2015: 18).

In Deutschland, Österreich und der Schweiz beeinflusst die politische Grundorientierung die Einstellungen zu sozialer (sprich: vor allem finanzieller) Ungleichheit: Konservative und Liberale akzeptieren Privilegien für Wohlhabende und große Einkommensunterschiede eher und lehnen staatliche Umverteilung eher $\mathrm{ab}$ als Sozialdemokraten und Linke (Haller et al. 2015: 24). Eine Finanz(aus)bildung, die die Ungleichheitsdebatte tabuisiert, stützt damit angesichts der herrschenden finanziellen Verteilungsverhältnisse konservative und liberale Positionen; sie muss sich als politisch einseitig bezeichnen lassen. Mit den Grundsätzen einer pluralen Gesellschaft und eines weltanschaulich sowie politisch ausgewogenen Unterrichts ist das unvereinbar.

Auch die OECD positioniert sich einseitig und strebt danach, finanzielle Inklusion kontextfrei und unpolitisch aufzufassen und definiert sie als „process of promoting affordable, timely and adequate access to a wide range of regulated financial products and services and broadening their use by all segments of society" (OECD 2016: 54). Je mehr Produkte des Finanzmarkts Befragte kennen (Zahlungsverkehr, Sparen, Altersvorsorge, Kredit, Versicherung) und je mehr solcher Produkte sie halten oder wählen, desto höher ist ihr Inklusionsgrad. Inklusion meint also Integration als Kunden/in in die Märkte für Finanzdienstleistungen und nicht etwa die angemessene Teilhabe am monetären Wohlstand der Gesellschaft.

Was kann man daran sehen? Ökonomische Bildung und Finanzerziehung sind ein Politikum. In beiden Feldern werden gesellschafts-, wirtschafts- und bildungspolitische Konflikte um die Deutungshoheit ausgetragen. Es geht darum, wer sein politischideologisches Framing im Schulfach, im Lehrplan, im Schulbuch, im Unterricht und in den Prüfungsaufgaben durchsetzen kann. Wem es gelingt, schon in der medialen Orientierungsphase eines Themas wie ökonomische Bildung oder Finanzbildung seinen eigenen Frame zu etablieren, der kann davon in der
Routinephase der medialen Berichterstattung profitieren (Scheufele 2003: 91-106). Die schulische Finanzausbildung soll den Boden dafür bereiten. Empirisch deutet vieles darauf hin, dass der Erfolg hier vorwiegend aufseiten der Privatwirtschaft und ihrer Verbände liegt. Sie werden jedenfalls stark unterstützt von gleichgesinnten Medien und ihren Eigentümer/ innen, von neoliberal inspirierten Schlüsselakteur/innen aus allen Parteien und nicht zuletzt auch aus der Wirtschaftsdidaktik.

\section{Bildung als Freiheit im Denken und Handeln}

Diese Auseinandersetzungen halten an und verlangen höchste Aufmerksamkeit. Sie beziehen sich auf wirtschaftliche Glaubenssätze, Überzeugungen und Politiken, Kapitalismus, Marktwirtschaft, Sozialstaat und kollektive sowie individuelle Verwirklichungschancen, auf das Verhältnis von Demokratie und Wirtschaft, genauer: Finanzwirtschaft, auf die Grundsätze von politischer Rahmensetzung, Steuerung und Gegensteuerung, auf wirtschaftlich relevante Werte, Grundorientierungen und Vorstellungen vom „Guten Leben“, auf Verständnisse von Wachstum, Entwicklung und Nachhaltigkeit, auf das Bild von Unternehmer/innen, Investor/innen, Rentiers und abhängig Beschäftigten, auf Ungleichheit, Machtasymmetrie und Verteilungspolitik. Nicht zuletzt, sondern vor allem geht es auch um Durchsetzung und Absicherung von finanziellen und politischen Interessen.

Den skizzierten Konflikten um sozioökonomische und finanzielle Bildung liegt also eine grundlegende und mehrdimensionale Kontroverse zugrunde, die politische, sozialpolitische, wirtschaftspolitische, interessenpolitische, wissenschaftspolitische, ideologische und weltanschauliche Dimensionen hat. Zur Debatte steht die inhaltliche Qualität von Bildung in der wirtschaftlichen Domäne, es geht um reflektierte Bildung versus funktionalistisches Lernen. Zugespitzt formuliert steht Aufklärung gegen Anpassung, Pluralität im Denken versus Monopol für eine Denkweise.

In diesem Konfliktfeld steht die sozioökonomische Bildung für Bildung, für Bildung als Freiheit und für Bildung als Chance in Alternativen und alternativ denken zu lernen. Die Prinzipien sozioökonomischer Bildung bestärken die Befreiung des Denkens über Wirtschaft in der Gesellschaft durch Kritik und Ideologiekritik, Pluralität und Kontroversität, Subjekt- und Problemorientierung sowie durch die soziopolitische und historische Einbettung wirtschaftlicher Phänomene und Probleme.

Freiheit der Person verlangt Freiheit im Denken, Freiheit in der Weltorientierung, Freiheit im Wirt- 
schaftsdenken, Freiheit in den persönlichen Wirtschaftsphilosophien, Freiheit der Leitbilder für eine bessere Wirtschaftswelt. Das schließt die Freiheit zum Aufbegehren gegen herrschende Verhältnisse und zum Aufstehen für eine bessere Welt ebenso ein wie die Freiheit zu Akzeptanz und Passivität. Denn Freiheit im Denken mündet in Freiheit im Handeln und im Nichthandeln.

Aber Freiheit im Denken setzt das Denken in Alternativen notwendigerweise voraus. Schule und Unterricht müssen Freiheit im ökonomischen, finanziellen und politischen Denken garantieren und fördern. Dazu tragen die sozialwissenschaftliche und die politische Pluralität wesentlich bei. Sozioökonomische Bildung ist deshalb wissenschaftsorientierte, nicht wirtschaftsorientierte Bildung. Auch deshalb ist sie die bessere ökonomische Bildung.

\section{Dank}

Die Veröffentlichung dieses Beitrags wurde vom Open Access Publikationsfonds der Universität Bielefeld unterstützt

\section{Literatur}

Akerlof, G. A. \& R. J. Shiller (2009): Animal spirits. Wie Wirtschaft wirklich funktioniert. Frankfurt am Main: Campus-Verlag.

Allmendinger, J., K. Jahn, M. Promberger, B. Schels \& S. Stuth (2018): Prekäre Beschäftigung und unsichere Haushaltslagen im Lebensverlauf. Gibt es in Deutschland ein verfestigtes Prekariat? In: WSI-Mitteilungen 71 (4). S. 259-269.

Aprea, C., E. Wuttke, K. Breuer, N. K. Koh, P. Davies, B. Greimel-Fuhrmann et al. (Hrsg.) (2016): International Handbook of Financial Literacy. Singapore: Springer.

Ariely, D. (2008): Denken hilft zwar, nützt aber nichts. Warum wir immer wieder unvernünftige Entscheidungen treffen. München: Droemer.

Arthur, C. (2012): Financial Literacy Education for Citizens. What kind of responsibility, equality and engagement? In: Citizenship, Social and Economics Education 11 (3). S. 163-176.

Becker, G. S. (1993): Der ökonomische Ansatz zur Erklärung menschlichen Verhaltens. 2. Aufl. Tübingen: Mohr.

Bieri, P. (2008): Wie wäre es, gebildet zu sein? In: Göppel, R., V. Lenhart, T. Rihm, B. Schön \& V. StrittmatteHaubold (Hrsg.), Bildung ist mehr. Potentiale über PISA hinaus. Heidelberg: Mattes. S. 13-21.

Blank, F., C. Logeay, E. Türk, J. Wöss \& R. Zwiener (2016): Alterssicherung in Deutschland und Österreich: Vom Nachbarn lernen? (WSI-Report Nr. 27), Düsseldorf.
Corneo, G. (2018): Ein Staatsfonds, der eine soziale Dividende finanziert. In: Perspektiven der Wirtschaftspolitik 19 (2). S. 94-109.

Deutsches Aktieninstitut (2013): Dienende Funktion der Kapitalmärkte erhalten und ausbauen - programmatische Anregungen für die künftige Bundesregierung. Positionspapier des Deutschen Aktieninstituts e.V., 23. Oktober 2013, Frankfurt am Main.

Deutschmann, C. (2013): Finanzialisierung als Hegemonie der Rentiers: eine soziologische Analyse. In: Pahl, H. \& J. Sparsam (Hrsg.): Wirtschaftswissenschaft als Oikodizee? Diskussionen im Anschluss an Joseph Vogls Gespenst des Kapitals. Wiesbaden: Springer Fachmedien; Wiesbaden: Springer VS.

Dullien, S. (2013): Umbau der Finanzmärkte. Übermäßiges Vertrauen in Marktrationalität hält an. In: Wirtschaftsdienst 93 (1). S. 23-29.

Engartner, T. (2016): Rendite statt Rente - oder: Die Privatisierung der Altersvorsorge. In: Gesellschaft, Wirtschaft, Politik 65 (4). S. 419-426.

Fessler, P. \& M. Schürz (2017): Länderunterschiede in der Vermögensungleichheit in Europa. In: Wirtschaft und Gesellschaft 43 (1). S. 41-64.

Ferschli, B., J. Kapeller, B. Schütz \& R. Wildauer (2017): Bestände und Konzentration privater Vermögen in Österreich. Simulation, Korrektur und Besteuerung. (ICAE Working Paper Series, 72). Linz.

Fohrmann, O. (2016): Im Spiegel des Geldes. Bildung und Identität in Zeiten der Ökonomisierung. Bielefeld: transcript.

Fonds professionell Online (2017): Sparkassentag 2017: Mehr Finanzbildung und weniger Regulatorik. http:// www.fondsprofessionell.at/news/unternehmen/headline/ sparkassentag-2017-mehr-finanzbildung-und-wenigerregulatorik-137578/ (08.08.2018)

Fridrich, C. (2015): Ökonomische Bildung im Trägerfach Geographie und Wirtschaftskunde zwischen Lebensweltorientierung und ökonomistischen Zugängen. Teilergebnisse einer empirischen Studie. In: Mitteilungen der Österreichischen Geographischen Gesellschaft 157. S. 277-300.

Fridrich, C. (2018): Sozioökonomische Bildung an allgemeinbildenden Schulen der Sekundarstufe I und II in Österreich. Entwicklungslinien und Plädoyer für das Integrationsfach Geographie und Wirtschaftskunde. In: Engartner, T., C. Fridrich \& S. Graupe (Hrsg.): Sozioökonomische Bildung und Wissenschaft. Entwicklungslinien und Perspektiven. Wiesbaden: Springer VS. S. 81-108.

Fridrich, C. \& M. Hofmann-Schneller (2017): Positionspapier „Sozioökonomische Bildung“. In: GW-Unterricht 145. S. 54-57.

Geisberger, Tamara (2017): Niedriglohnbeschäftigung in Österreich 2014: geringe Dynamik - verfestigte Strukturen. In: Statistische Nachrichten 11. S. 924-939.

Gökbudak, M. \& R. Hedtke (2017): 17 Minuten Politik, 20 Sekunden Redezeit. Daten zum Politikunterricht in der 
Sekundarstufe I in Nordrhein-Westfalen (Didaktik der Sozialwissenschaften, Working Papers Nr. 6), Bielefeld.

Grabka, M. M. (2014): Ungleichheit in Deutschland Langfristige Trends, Wendepunkte. In: Sozialer Fortschritt 63 (12). S. 301-307.

Grabka, M. M. \& C. Westermeier (2014): Anhaltend hohe Vermögensungleichheit in Deutschland. In: DIW-Wochenbericht 81 (9). S. 151-164.

Greimel-Fuhrmann, B. (2012/13): “Don't know much about economics and business". Economic und financial literacy als wesentliche, jedoch vernachlässigte Bildungsziele. In: WissenPlus 4. I-VIII.

Greimel-Fuhrmann, B., M. Silgoner, R. Weber \& M. Taborsky (2016): Financial Literacy in Austria. In: Aprea, C., E. Wuttke, K. Breuer, N. K. Koh, P. Davies, B. Greimel-Fuhrmann et al. (Hrsg.): International Handbook of Financial Literacy. Singapore: Springer. S. 251-262.

Groh-Samberg, O. (2014): No Way Out - Dimensionen und Trends der Verfestigung der Armut in Deutschland. In: Sozialer Fortschritt 63 (12), S. 307-315.

Haller, M., A. Eder \& B. M. Kmet (2015): Drei Wege zur Zähmung des Kapitalismus. In: Österreichische Zeitschrift für Soziologie 40 (1). S. 1-31.

Hedtke, R. (2015): Sozioökonomische Bildung als Innovation durch Tradition. In: GW-Unterricht 140. S. 18-38.

Hedtke, R. (2018): Das Sozioökonomische Curriculum und die Grundlagen der sozioökonomischen Bildung. Frankfurt am Main: Wochenschau Verlag (In Druck).

Hippe, T. \& Hedtke, R. (2006): Kapitalgedeckte Rentensysteme. Wissenschaftsorientierung, Pluralismus und Kontroversität. In: Seeber, G. (Hrsg.): Die Zukunft der sozialen Sicherung - Herausforderungen für die ökonomische Bildung. Bergisch Gladbach: Thomas Hobein. S. 27-50.

Kaminski, H. (Hrsg.) (2010): Praxis Wirtschaft. Gesamtband. Braunschweig: Westermann.

Kaminski, H. (2012): Die Wirtschaftsordnung als fachdidaktischer Reflexionsgegenstand der ökonomischen Bildung. In: Schuhen, M., M. Wohlgemuth \& C. Müller (Hrsg.), Ökonomische Bildung und Wirtschaftsordnung. Stuttgart: Lucius \& Lucius.

Kaminski, H. \& S. Friebel (2012): Arbeitspapier „Finanzielle Allgemeinbildung als Bestandteil der ökonomischen Bildung“. Oldenburg: Institut für Ökonomische Bildung.

Kepplinger, H. M. (2010): Medieneffekte. Wiesbaden: VS Verlag für Sozialwissenschaften.

Kirchgässner, G. (2008): Homo oeconomicus. Das ökonomische Modell individuellen Verhaltens und seine Anwendung in den Wirtschafts- und Sozialwissenschaften (3. Aufl.). Tübingen: Mohr Siebeck.

KMK (2013/1972): Vereinbarung zur Gestaltung der gymnasialen Oberstufe in der Sekundarstufe II. Beschluss der Kultusministerkonferenz vom 07.07.1972 i.d.F. vom 06.06.2013 (Sekretariat der Ständigen Konferenz der Kultusminister der Länder in der Bundesrepublik Deutschland, Hrsg.), o. O. [Bonn].
KMK (2014/1993): Vereinbarung über die Bildungsgänge im Sekundarbereich I. Beschluss der Kultusministerkonferenz vom 03.12.1993 i.d.F. vom 25.09.2014 (Sekretariat der Ständigen Konferenz der Kultusminister der Länder in der Bundesrepublik Deutschland, Hrsg.), o. O. [Bonn].

Knabe, A. \& J. Weimann (2017): Die Deutschlandrente: Ein Konzept zur Stärkung der kapitalgedeckten Altersvorsorge. In: ifo Schnelldienst 70 (18). S. 25-33.

Koch, T. \& T. Zerback (2011): Wiederholung und Wahrheit. Wie die mehrmalige Präsentation politischer Slogans die Einschätzung ihrer Glaubwürdigkeit beeinflusst. In: Medien \& Kommunikationswissenschaft 59 (4). S. 487-504.

Koch, M. \& S. Friebel (2015): Unterrichtseinheit „Finanzielle Allgemeinbildung“ (3. Aufl.). o. O. (Oldenburg): Institut für Ökonomische Bildung.

Kohlert, D. \& A. Oehler (2009): Scheitern Finanzdienstleistungen am Verbraucher? Eine theoretische Analyse rationalen Verbraucherverhaltens im Rahmen des Anlageberatungsprozesses. In: Vierteljahreshefte zur Wirtschaftsforschung 78 (3). S. 81-95.

Koller, H.-C. (2018): Bildung anders denken. Einführung in die Theorie transformatorischer Bildungsprozesse (2. Aufl.). Stuttgart: Kohlhammer Verlag.

Kranzinger, S. (2016): Eine Analyse des Prozesses der Vermögensakkumulation anhand des Konzeptes der Pfadtheorie. In: Wirtschaft und Gesellschaft 42 (1), S. 131-160.

Lemmer, B. (2017): Zinseszins wird oft nicht verstanden. http://diepresse.com/home/meingeld/aktien/5271796/ Zinseszins-wird-oft-nicht-verstanden (08.08.2018)

Masciandaro, D. (Hrsg.) (2004): Global Financial Crime. Terrorism, Money Laundering and Offshore Centres. Florence: Taylor and Francis.

Mayntz, R. (2012): Die transnationale Ordnung globalisierter Finanzmärkte. Was lehrt uns die Krise? In: Soeffner, H.-G. (Hrsg.): Transnationale Vergesellschaftungen. Verhandlungen des 35. Kongresses der Deutschen Gesellschaft für Soziologie in Frankfurt am Main 2010. Wiesbaden: Springer VS. S. 389-400.

Mayntz, R. (2014): Die Finanzmarktkrise im Licht einer Theorie funktioneller Differenzierung. In: KZfSS Kölner Zeitschrift für Soziologie und Sozialpsychologie 66 (1). S. 1-19.

Mertens, D. \& R. Meyer-Eppler (2014): PensionsfondsKapitalismus und privatisierter Keynesianismus. Zur Finanzialisierung der Haushalte. In: Heires, M. \& A. Nölke (Hrsg.): Politische Ökonomie der Finanzialisierung. Wiesbaden: Springer Fachmedien Wiesbaden. S. 259-274.

Mönter, L., S. Lippert \& A. Gorges (2016): Gemachte Armutsräume? Implikationen bei der Behandlung von Entwicklungsdisparitäten im geographischen Unterricht, vom Schulbuch zur Schülervorstellung. In: GW-Unterricht 142/143. S. 71-79.

Nullmeier, F. (2015): Einstürzende Neubauten - Statikprobleme im Säulenmodell der Alterssicherung. In: Sozialer Fortschritt 64 (8). S. 196-202. 
OECD (2015): 2015 OECD/INFE Toolkit for Measuring Financial Literacy and Financial Inclusion, Paris.

OECD (2016): OECD/INFE International Survey of Adult Financial Literacy Competencies, o. O. [Paris].

OECD (2017): G20/OECD INFE Report on Adult Financial Literacy in G20 Countries, o. O. [Paris].

Oehler, A. \& S. Wendt (2017): Good Consumer Information. The Information Paradigm at its (Dead) End? In: Journal of Consumer Policy 40 (2). S. 179-191.

Ötsch, W., S. Pühringer \& K. Hirte (2018): Netzwerke des Marktes. Ordoliberalismus als Politische Ökonomie. Wiesbaden: Springer VS.

Platt, S. (2015): Criminal Capital. How the Finance Industry Facilitates Crime. Basingstoke, Hampshire, New York: Palgrave Macmillan.

Reichenbach, R. (2007): Kompetenzen und Insuffizienzen. Bemerkungen zur neuen Lust, schulische Bildung zu kontrollieren, zu steuern und zu messen. Vortrag an der Aarg. Kantonalen Lehrerinnen- und Lehrerkonferenz. https://www.ife.uzh.ch/dam/jcr:44438f77-f8ed-4844866f-143454affb83/Kompetenzen\%20und\%20Insuffizienzen.pdf (08.08.2018)

Reifner, U. (2011): Finanzielle Allgemeinbildung und ökonomische Bildung. In: Retzmann, T. (Hrsg): Finanzielle Bildung in der Schule. Mündige Verbraucher durch Konsumentenbildung. Schwalbach/Ts.: Wochenschau. S. 9-30.

Reifner, U. (2017): Soziologie des Geldes. Heuristik und Mythos (Das Geld, Bd. 2). Wiesbaden: Springer VS.

Remmele, B. (2016): Financial Literacy and Financial Incomprehensibility. In: Aprea, E. Wuttke, K. Breuer, N. K. Koh, P. Davies, B. Greimel-Fuhrmann et al. (Hrsg.): International handbook of financial literacy. Singapore: Springer. S. 9-24.

Retzmann, T. (2016): Die Schule ist ein wirtschaftsfreier Raum. Leserbrief. In: Finanztest 5, S. 6.

Retzmann, T. \& G. Seeber (2016): Financial Education in General Education Schools: A Competence Model. In: Aprea, C., E. Wuttke, K. Breuer, N. K. Koh, P. Davies, B. Greimel-Fuhrmann et al. (Hrsg.): International Handbook of Financial Literacy. Singapore: Springer. S. 39-56.

Scheufele, B. (2003): Frames - Framing - Framing-Effekte. Theoretische und methodische Grundlegung des Framing-Ansatzes sowie empirische Befunde zur Nachrichtenproduktion. Wiesbaden: Westdeutscher Verlag.
Schimank, U. (2006): Rationalitätsfiktionen in der Entscheidungsgesellschaft. In: Tänzler, D., H. Knoblauch \& H.-G. Soeffner (Hrsg.): Zur Kritik der Wissensgesellschaft. Konstanz: UVK Verlagsgesellschaft. S. 57-81.

Stein, U. (2014): Erklärungsansätze für die Ungleichheitsentwicklung in Deutschland. In: Sozialer Fortschritt 63 (12). S. 315-321.

Stieger, S. \& T. Jekel (2018): Vermessene ökonomische Bildung? In: GW-Unterricht 150. S. 5-19.

Stiftung Warentest. (2015): Schulprojekt zur ökonomischen Bildung. https://www.test.de/presse/pressemitteilungen/Schulprojekt-zur-oekonomischen-Bildung-Jetztfuer-das-Schuljahr-20152016-bewerben-4835078-0/ (08.08.2018)

Weber, B. (2010): Finanzbildungsbürgertum und die Finanzialisierung des Alltags. In: PROKLA Zeitschrift für kritische Sozialwissenschaft 160. S. 377-393.

Weber, B. (2018): Ökonomische Bildung in der Sekundarstufe I Eine curriculare Analyse ihrer Relevanz, Inhalte und Kompetenzerwartungen. Frankfurt am Main: Wochenschau Verlag.

Wehling, E. (2016): Politisches Framing. Wie eine Nation sich ihr Denken einredet - und daraus Politik macht. Köln: Herbert von Halem Verlag.

Wendt, B., M. B. Klöckner, S. Pommrenke \& M. Walter (Hrsg.) (2016): Wie Eliten Macht organisieren. Bilderberg \& Co.: Lobbying, Thinktanks und Mediennetzwerke. Hamburg: VSA Verlag.

Westermeier, C. \& M. M. Grabka (2015): Große statistische Unsicherheit beim Anteil der Top-Vermögenden in Deutschland. In: DIW-Wochenbericht 82 (7). S. 123-133.

Willis, L. E. (2018): Finanzinformierte Bürger_innen, bürgerbestimmtes Finanzsystem. Ein Essay aus Anlass des International Handbook of Financial Literacy. In: Journal of Social Science Education 17 (1). S. 77-88.

Young, B. (2014): Finanzialisierung, Neoliberalismus und der deutsche Ordoliberalismus in der EU-Krisenbewältigung. In: M. Heires \& Nölke A. (Hrsg.): Politische Ökonomie der Finanzialisierung. Wiesbaden: Springer Fachmedien. S. 63-78. 